\title{
Cartaphilus
}

Revista de investigación y crítica estética

ENTRONIZAR LA NADERÍA: ANALOGÍA, IRONÍA

Y METAIRONÍA EN EL READY-MADE DUCHAMPIANO SEGÚN OCTAVIO PAZ

ENTHRONING THE NOTHING: ANALOGY, IRONY

AND META-IRONY IN DUCHAMP'S READY-MADES ACCORDING TO OCTAVIO PAZ

\section{MARCELO URRALBURU}

UNIVERSIDAD DE MURCIA

Resumen: En este artículo vamos a desarrollar una serie de conceptos críticos de la obra ensayística de Octavio Paz, concretamente, los de analogía e ironía, de raigambre claramente romántica y simbolista, y el metaironía, conceptualizado específicamente por el autor para explicar un fenómeno estético propio de las vanguardias artísticas. Sin embargo, no nos dedicaremos a todos los artistas que renovaron las formas de creación cultural a principios del siglo $\mathrm{XX}$, sino que nos detendremos en la obra temprana de un figura que interesó especialmente a Octavio Paz: Marcel Duchamp. De esta manera, explicamos la evolución y aplicación de estos conceptos a través de diferentes reflexiones y autores de interés. Observaremos que en el fondo de estas reflexiones se encuentran las claves de la modernidad poética, según era entendida por el poeta mexicano, y que se manifestaba en una trayectoria cultural que culminaba o alcanzaba la plenitud, precisamente, con la obra de Duchamp.

Abstract: In this paper, we are going to develop a series of critical concepts from Octavio Paz's essay works, specifically, those of analogy and irony, of clearly romantic and symbolist roots, and meta-irony, particularly conceptualized by the author to explain an aesthetic phenomenon of the Avant-garde. However, we will not devote ourselves to all the artists who renewed the forms of cultural creation at the beginning of the 20th century, but we will stop at the early work of a figure that was specially interesting to Octavio Paz: Marcel Duchamp. In this way, we are going to explain the evolution and application of these concepts through different reflections and authors of interest. We will observe that at the bottom of these reflections are found the keys of poetic 
modernity as understood by the Mexican poet, and that it manifested itself in a cultural trajectory that culminated or reached fullness, precisely, with Duchamp's works.

Palabras clave: Octavio Paz; Marcel Du- champ; metaironía; modernidad; readymade.

Keywords: Octavio Paz; Marcel Duchamp; meta-irony; modernity; readymade.

\section{INTRODUCCIÓN}

El lugar que ocupa Marcel Duchamp en el ensayismo de Octavio Paz resulta ser una cuestión problemática, en parte por la complejidad de las ideas que se introducen en estos textos, y en parte por el enigma que aún se extiende por sobre su diálogo artístico y poético. Octavio Paz consideraba al francés el resultado de una tradición de la ruptura; la consecuencia natural de una evolución hasta cierto punto programada. Cada una de sus obras constituyó un mazazo contra la convención literaria, cuestionando incluso la innovación que se presuponía en las nuevas técnicas pictóricas y los nuevos lenguajes artísticos. El crítico supo describir esta realidad con una minuciosidad pasmosa, pero ante todo descubrió una trama de incógnitas que aún no termina de cerrarse.

Esta investigación tiene por objetivo contraponer algunas de las teorías poéticas y creativas de Octavio Paz a su concepción de la modernidad y, en concreto, qué lugar ocupa para él la figura de Marcel Duchamp en esta trama de continuidades y rupturas. Dónde reside su originalidad y a qué se debe su interés. Para ello resulta necesario, en primer lugar, esclarecer ciertas cuestiones textuales relativas a los textos que el mexicano dedicó al artista, pues se trata de unas ideas que fueron sucesivamente ampliadas, corregidas, matizadas, en distintas fases de escritura: homenajes, encargos, revisiones. Los particulares procedimientos textuales de Octavio Paz complican tanto la lectura de sus textos, que se superponen en un dilatado palimpsesto, como en su interpretación de conjunto. Tanto es así que existen cuantiosas diferencias entre las distintas ediciones, siendo la definitiva y más completa la contenida en la edición que él mismo preparó de sus obras completas para el Círculo de Lectores: Los privilegios de la vista. Arte moderno universal I, donde encontramos la versión definitiva del ensayo sobre Duchamp: Apariencia desnuda (1992).

Actualmente, no tenemos constancia del primer encuentro entre ambas figuras, así como no podemos sino especular sobre el interés inicial que la obra de Duchamp despertó en Octavio Paz, pero sí sabemos de una comunicación epistolar entre ellos en los años en que este fue embajador de México en la India. Una parte de esta relación ha sido documentada por Monique Fong, quien sería la traductora del poeta al francés y quien mediaría entre ambos personajes (Fong, 2014). En cuanto al texto propiamente, una primera aproximación crítica sería el ensayo Marcel Duchamp o El castillo de la pureza, escrito en Nueva Delhi 
y datado del 25 de octubre de 1966 -corregido posteriormente en Ciudad de México hacia el 20 de marzo de 1976. Fue publicado por el editor Vicente Rojo con un formato de "libro-maleta" a modo de homenaje a La Boîte-en-valise (1941) de Duchamp; esta edición limitada y rápidamente agotada incluía una serie de reproducciones y láminas de la obra del artista, un retrato del mismo, el texto de Paz y una selección de textos de la Caja verde (1934). Este texto pudo ser leído por Marcel y Teeny Duchamp, su esposa, en su vivienda de Nueva York gracias a la traducción de Monique Fong (2014: 22); en él, Paz aportaba su particular lectura de la figura y la obra del francés, una descripción detallada de los elementos constitutivos del Gran vidrio (1915-1923) y una explicación de su meticuloso funcionamiento a partir de las notas contenidas en la Caja verde y de otros críticos de arte relevantes.

Octavio Paz publicó un segundo ensayo con motivo de una gran exposición retrospectiva de la obra de Marcel Duchamp, celebrada por el Museo de Arte de Filadelfia y el Museo de Arte Moderno de Nueva York en el otoño de 1973, a petición de ambas instituciones. Si bien en ambos textos se aborda la totalidad de la obra duchampiana, podemos afirmar que el primero está mayormente dedicado al Gran vidrio, nombre con el que la crítica suele referirse a $L a$ novia puesta al desnudo por sus solteros, aun... ${ }^{1}$, y el segundo sería una mirada retrospectiva cuya intención consistiría, precisamente, en dotar de una coherencia temática y artística al conjunto de piezas; sobre todo a la luz de Dados: 1. - La Cascada, 2. El Gas de alumbrado (1969), obra póstuma a la que se dedicó Duchamp a lo largo de veinte años (de 1946 a 1966), tiempo en que hizo ver que había abandonado el arte. Este segundo ensayo tiene por título * water writes always in * plural ${ }^{2}$ en alusión a una frase que apareció en el primer artículo publicado en inglés por Duchamp: The (Nueva York, 1915), en el cual la palabra the ('el' o 'la') era sistemáticamente sustituida por un asterisco $\left({ }^{*}\right)$.

Este texto, escrito ya en Ciudad de México hacia el 27 de diciembre de 1972, fue corregido y ampliado el 3 de agosto de 1976. Por supuesto, la corrección a la que Paz sometió ambos textos en el año de 1976 tiene como consecuencia que entre las primeras ediciones de este texto y la versión última existan cuantiosas diferencias, tanto en el estilo como en el contenido y la estructura del ensayo. En la "Advertencia" que precede a la edición definitiva, también escrita en 1976, Paz se preocupa por destacar tales diferencias y de aclarar el origen y el propósito de sus escritos:

Al presentar esta nueva edición no solo corregí, aquí y allá, el primer estudio sino que le añadí más de treinta páginas en las que procuro describir todos los elementos del Gran vidrio, sus funciones y las relaciones que los unen. También

\footnotetext{
${ }^{1}$ La traducción del original La Mariée mise à un par ses célibataires, même es propuesta por el propio Paz en este ensayo con la consecuente matización en torno a su significado.

2 * agua escribe siempre en * plural, según traduce el propio Octavio Paz.
} 
revisé el segundo ensayo y agregué otras cuarenta páginas (Paz, 1992b: 129).

Un estudio filológico pormenorizado de las diferencias entre las múltiples ediciones de Apariencia desnuda supondría de gran interés para comprender al detalle los cambios de estilo, pensamiento y revisión que sufrió el ensayismo de Octavio Paz. Sin duda, muchos de ellos tienen la importancia de revelar cómo se transformó la manera en que nuestro autor entendía la modernidad y su mirada sobre la obra de Duchamp; también cómo quiso ordenar y dar coherencia al conjunto de sus textos, pues la progresiva ampliación del texto incluía referencias a otros textos nuevos sobre otros aspectos del arte del siglo XX y de la crisis de la modernidad. Sin embargo, a pesar del interés que ello guarda para la crítica, las exigencias de un estudio de tales características implicarían una profundidad y una extensión incompatibles con el análisis que nos ocupa; más interesado, en cambio, en desentrañar las características del pensamiento paciano de cara a la crisis de la modernidad y su contemplación de la obra de Marcel Duchamp como un gozne o engranaje fundamental dentro de dicha crisis.

\section{ANALOGÍA, IRONÍA Y METAIRONÍA}

El espíritu crítico y la ironía son una constante en la obra de Marcel Duchamp. No podemos dibujar una línea divisoria que escinda una parte y otra, pero a lo largo de este epígrafe nos centraremos en el giro dialéctico que el artista francés introdujo en la modernidad de las vanguardias. A propósito de la lógica dialéctica que rige la modernidad, Octavio Paz reconoce en el arte de Duchamp un giro crítico que lo distancia del resto de artistas de su tiempo: la metaironía. Para explicar dicho fenómeno desarrollamos nuestro análisis en dos direcciones: en primer lugar, el origen del concepto de ironía tal y como lo registra el autor mexicano en varios de sus ensayos, y a través de su conceptualización en el Romanticismo alemán; $y$, en segundo término, cómo dicho concepto se materializa en una de las grandes revoluciones de la historia del arte: los ready-mades conforme son teorizados por Paz, ya que la crítica ha manifestado grandes diferencias sobre esta cuestión. Así pues, procederemos a la revisión y reconstrucción del concepto de metaironía a través de los de analogía y de ironía desde el Romanticismo.

La revolución romántica trajo consigo una nueva concepción del universo como una entidad heteróclita y cambiante, regida por unas leyes a las que el filósofo aspiraba mediante el uso de la Razón - tal era el objeto de pensamiento de la filosofía idealista. Dichas leyes, además, en su regimiento regular y cíclico seguirían un orden o sentido universal constituido por el ritmo. De esta manera, el universo era entendido como un texto o tejido de signos cuyo movimiento rotativo está subordinado a unas leyes rítmicas y orgánicas que, a su vez, rigen también la vida del hombre y el lenguaje. El artista, por su parte, recrea en su obra las leyes del cosmos y los signos que lo habitan mediante la analogía, un sistema 
de correspondencias que traducen los signos del universo en signos lingüísticos o signos plásticos (Cervera, 2001: 104-123). Un buen ejemplo de esto, por ejemplo, se encontraría en la novela de Novalis Los discípulos de Sais, donde hallamos la siguiente metáfora del poeta a través de la figura del Maestro:

Pronto advirtió las combinaciones que unían todas las cosas, las similitudes, las coincidencias. A poco, ya no vio nada aisladamente. Las percepciones de sus sentidos se agolpaban en grandes y variadas imágenes. Oía, veía, tocaba y pensaba a un tiempo. Se complacía en unir cosas dispares. Ora las estrellas parecíanle hombres, ora los hombres parecíanle estrellas; las piedras, animales; y las nubes, plantas. Jugaba con las fuerzas y los fenómenos (Novalis, 1988: 29).

La sinestesia y la multiplicidad de lenguajes artísticos propugnada por el modernismo hispanoamericano habrían sido, en parte, heredadas de la cosmovisión romántica a través del simbolismo. Este planteamiento es fundamental para comprender la actitud crítica que asume Octavio Paz con respecto del arte, tomada en gran medida del pensamiento de Charles Baudelaire ${ }^{3}$. Como consecuencia de dicha concepción, la labor artística consistiría en la lectura del texto universal y su traducción en un lenguaje o sistema de signos cerrado y aprehensible, mientras que la labor crítica sería la traducción de la obra de arte en un código lingüístico con el fin de explicarla. Cada obra sería representación del mundo y un mundo en sí misma: traducción y metáfora. Todo estaría regido por un sistema de correspondencias sígnicas. Por ello, según se explica en Los hijos del limo (1974), la analogía tiene por consecuencia que el mundo ya no sea un "teatro regido por el azar y el capricho" sino que "lo gobiernan el ritmo y sus repeticiones y conjunciones"; es decir: "es un teatro hecho de acordes y reuniones en el que todas las excepciones, inclusive la de ser hombre, encuentran su doble y su correspondencia" (1999: 476).

¿Cómo surge la ironía, entonces, si la obra de arte es una reproducción del orden cósmico? Sobre la contradicción inmanente a la naturaleza y al hombre debemos acudir a "Sobre el modo de proceder el espíritu poético", ensayo de Friedrich Hölderlin (1770-1843), donde el poeta expuso sus consideraciones sobre la mutabilidad del ser y la libertad del hombre. Hölderlin encontraría una tensión entre el cambio y la identidad material, esto es, la mutabilidad y la inmutabilidad del ser - antigua querella desde los filósofos presocráticos: Parménides y Heráclito. También la humanidad estaría condenada a una "contradictoria condición intermedia"; aquella que existe entre la conexión natural con el mundo - por ejemplo: la propia de la niñez - y la conexión natural con libre elección -el distanciamiento que permite la abstracción crítica del mundo natural, ya en la madurez- (Hölderlin, 2014: 65). En lo que se traduce dicha tensión entre

\footnotetext{
${ }^{3}$ Sobre esta cuestión es especialmente relevante el ensayo de Octavio Paz titulado: "Presencia y presente: Baudelaire crítico de arte" (En El signo y el garabato, 1973).
} 
cambio e identidad material, como bien señala Manuel Carbonell en la edición que lleva a cabo de este ensayo, es en la confrontación entre una concepción del tiempo "dinámica, cíclica, y otra lineal, progresiva" (2014: 7).

No nos interesa profundizar mucho más en estos aspectos, si bien estas apreciaciones sirven para mostrar cómo la visión cosmológica y artística del Romanticismo integraba la tensión contradictoria como la esencia misma de la naturaleza y del hombre. Esta contradicción, esta divergencia de sentidos y de formas en el universo, es la misma que existe entre el doble sentimiento de pertenencia y de extrañeza que experimenta el ser humano con respecto al medio natural que habita. Esta contradicción se resuelve en la ironía (Ventura Ramos, 2015).

No hay que olvidar, por otro lado, que en el fenómeno romántico se encuentran los inicios de la modernidad. Si volvemos sobre nuestros pasos para retomar la citada querella entre los antiguos y los modernos, observaremos la doble realidad o la contradicción inherente al concepto de lo moderno según Baudelaire: "la modernidad es lo transitorio, lo fugitivo, lo contingente, la mitad del arte, cuya otra mitad es lo eterno y lo inmutable" (1995: 92). También en los movimientos estéticos se encuentra la tensión irónica entre la constancia y la ruptura; entre su deseo de perduración y su consciencia de finitud. A propósito de esta realidad, el propio Octavio Paz pronostica en La otra voz (1990) que "la Edad Moderna no tardará en ser la Antigüedad de mañana" (1999: 603). En otras palabras, la conciencia de pertenecer a una tradición concreta - ejercicio de abstracción- permite, al mismo tiempo, su crítica y su negación; es decir, genera, a su vez, un punto de fuga en el concepto de la analogía: la ironía como negación de la tradición. La tradición de la ruptura, en su conciencia como tradición, se sabe finita y condenada a la desaparición. Así como la analogía permite la correspondencia universal, la ironía supone una anulación del universo. Octavio Paz explicará así este fenómeno:

La analogía convierte a la ironía en una variación más del abanico de las semejanzas, pero la ironía desgarra el abanico. La ironía es la herida por la que se desangra la analogía; es la excepción, el accidente fatal, en el doble sentido del término: lo necesario y lo infausto. La ironía muestra que, si el universo es una escritura, cada traducción de esa escritura es distinta, y que el concierto de las correspondencias es un galimatías babélico. La palabra poética termina en un aullido o silencio: la ironía no es una palabra ni un discurso, sino el reverso de la palabra, la no-comunicación. El universo, dice la ironía, no es una escritura; si lo fuese, sus signos serían incomprensibles para el hombre porque en ella no figura la palabra muerte, y el hombre es mortal (1999: 484).

El mejor ejemplo de cómo funciona la ironía en términos poéticos y dialécticos será, para Paz, el caso del posmodernismo hispanoamericano. Como es sabido, dicho movimiento recibe su nombre por ser la producción tardía o inme- 
diatamente posterior al movimiento modernista y, sin embargo, al mismo tiempo, constituye un nuevo estadio del mismo a través de una crítica de su decadencia. El posmodernismo es el primer movimiento hispanoamericano verdaderamente moderno porque tiene conciencia de mortalidad: "hace poesía con la crítica de la poesía". Determinados poetas modernistas acabaron cediendo ante formas coloquiales e introduciendo la ironía en su lenguaje: "la nota irónica, voluntariamente antipoética y por eso más intensamente poética, [...] aparece casi siempre asociada a la imagen de la muerte" (1999: 506).

Las apreciaciones de Octavio Paz, sin embargo, pueden resultar algo abstrusas fuera de su contexto. El desgarramiento que supone la ironía dentro de la diversidad heteróclita del universo, en realidad, puede ser explicado desde la concepción de la misma dentro de la filosofía de Søren Kierkegaard (1813-1855), y para tal fin tomaremos como referente la tesis que defenderá en 1841, titulada El concepto de ironía, con referencia constante a Sócrates. Previa a toda consideración, se hace necesaria la distinción que el propio autor plantea entre la ironía ejecutiva, aquella que se produce en un contexto comunicativo concreto y se puede describir en términos retóricos, y la ironía contemplativa, negatividad o contradicción que impregna la manera en que el sujeto piensa el mundo (Ballart, 1994: 100). Por supuesto, Paz se refirió a esta última concepción, cuyo origen se encuentra en el idealismo alemán, cuando aludía al giro metairónico en la obra de Duchamp - aunque cada obra de arte individual guardaría unas formas que, hasta cierto punto, podrían ser analizadas en términos retóricos.

Sin embargo, ¿qué es lo que realmente nos interesa sobre Kierkegaard y la ironía? No únicamente es importante para comprender la obra de Duchamp, la propia modernidad como la entiende Paz está condicionada por el gesto irónico como manifestación de la pasión crítica. Y, en este sentido, Paz no dista de Kierkegaard, sobre las diferencias que existen entre la duda y la ironía, cuando el danés afirma: with irony, on the other hand, the subject is always seeking to get outside the object, and this he attains by becoming conscious at every moment that the object has no reality ${ }^{4}$ (citado en Ballart, 1994: 101). Pere Ballart ha explicado esta descripción de la ironía como la negación de la realidad de los objetos, cuyo objetivo es trascenderlos, con suma precisión; las ideas invariantes que inciden en la filosofía de Kierkegaard son: "la visión privilegiada y superior del ironista; el relativismo, que disuelve las apariencias del mundo en puras paradojas, y el castigo de toda ingenuidad respecto a las posibilidades reales del entendimiento humano" (Ballart, 1994: 102).

Estas son algunas de las claves necesarias para entender, igualmente, la dialéctica de la crítica moderna en su vertiente irónica - fundamental sobre todo en el vanguardismo. Sin embargo, aún quedaría por explicar cuál es la función

\footnotetext{
4 "A través de la ironía, por otra parte, el sujeto está constantemente buscando cómo salir del objeto, y lo logra siendo consciente en todo momento de que el objeto no es real" (la traducción es mía).
} 
concreta de la ironía. La concepción filosófica de la ironía está inevitablemente vinculada al pensamiento escéptico; ya lo advertía el propio Paz cuando afirmaba que "el universo, dice la ironía, no es una escritura; si lo fuese, sus signos serían incomprensibles para el hombre" (1999: 484). Por su parte, Kierkegaard parece ser más explícito sobre el propósito del pensamiento y del lenguaje irónico. Para el danés, la ironía no plantearía una negación absoluta de la realidad, sino una liberación del pensamiento dogmático; buscaría la suspensión del juicio sobre la realidad. Sócrates se sirve en los diálogos platónicos de la ironía, precisamente, en la justa medida en que se sirve de la pregunta para mostrar las fallas del pensamiento del otro: mina su confianza al tiempo que mina la posibilidad misma de conocer. ¿Cuál es la finalidad de la pregunta, entonces, y dónde se encuentra el gesto irónico? Kierkegaard es del todo claro al respecto:

Se puede hacer una pregunta con la intención de conseguir una respuesta que tenga el contenido deseado, de forma que cuantas más preguntas se hagan, más profundidad y significado adquiera la respuesta; o se puede hacer una pregunta, no por el interés de conseguir una respuesta sino para extraer el contenido aparente con la pregunta y dejar solamente el vacío. El primer método presupone, naturalmente, el contenido, el segundo un vacío; el primero es especulativo, el segundo irónico (citado en Ballart, 1994: 103).

No hay que olvidar, sin embargo, que la ironía plantea una contradicción que, para Kierkegaard, deberá ser trascendida y "domada" con el fin de superar la negatividad y la vacuidad que postula (Ballart, 1994: 105). La solución que propone el danés será de índole teológico y a ella llegará a través de una subyugación del proceso irónico a sus propios valores cristianos. Pere Ballart resume muy acertadamente que, para Kierkegaard, "el potencial con que la ironía nos muestra la incapacidad y finitud de las cosas humanas" debe trocar "su signo en un valor positivo" y permitir a la humanidad "encontrar a Dios en el reconocimiento de sus propias flaquezas" (1994: 106-107). Esta faceta positiva de la ironía no resulta pertinente en lo que respecta a este trabajo por lo que no ahondaremos más allá de esta breve nota, necesaria para marcar distancias entre el concepto de ironía en la filosofía Kierkegaard y el pensamiento romántico y la ironía que describe Octavio Paz en la obra de Marcel Duchamp.

Las contradicciones de la modernidad, su progresión dialéctica mediante la crítica, favorecen la aparición de la ironía en la obra de arte. El propio sintagma "tradición de la ruptura" pone de manifiesto la importancia que en ella va a tener dicho fenómeno. La metaironía - según nos es presentada en Los hijos del limo- es el resultado de dicha contraposición de extremos opuestos: magia/política, antiguo/moderno, amor/humor y, en definitiva, arte/vida. Pero, de nuevo nos preguntamos, ¿cuál es el objetivo de dicho giro y cómo se manifiesta? Basta con acudir a las palabras de Octavio Paz: "el resultado es la metaironía, una suerte de suspensión del ánimo, un más allá de la afirmación y de la negación" (1999: 520). Desde luego, estas palabras se acercan bastante a la lectura de 
Kierkegaard: ante la pregunta, el vacío.

Por otra parte, del mismo modo que la ironía suponía un intento por superar al objeto y denunciar su vacuidad, la metaironía trasciende la dimensión artística y eleva su crítica - su denuncia - al acto creativo mismo. Durante el proceso, además, el espectador se ve envuelto en el gesto artístico: “operación circular de la metaironía: el acto de ver una obra de arte convertido en un acto de voyeurisme. Mirar no es una experiencia neutral: es una complicidad" (1999: 520). Duchamp entendía que el acto creativo no se reduce a la intervención del artista sobre la realidad, sino que se concluye en el momento de la contemplación por parte del espectador (Duchamp, 2016: 66). Su intento por desneutralizar la mirada del espectador, sin embargo, lo obligó a buscar la neutralidad en otra parte: en la obra artística reducida al vacío irónico. Esta es la condición irónica de la obra de Marcel Duchamp, según Octavio Paz. La atraviesa por entero y sin excepción, y en este epígrafe aplicaremos el análisis de la metaironía al caso concreto de sus ready-mades.

El castillo de la pureza comienza con una concisa y esquemática introducción a la figura y la obra de Duchamp, lo que sirve a Paz para poner en claro algunos conceptos básicos y ciertos comentarios sobre las vanguardias, aunque más adelante su crítica deslindará parte a parte cada una de las facetas del artista a través de sus obras. Primeramente se detendrá en los ready-mades, que le permite introducir conceptos transversales que luego reaparecerán y preservar su linealidad argumentativa.

Desde su aparición, estos curiosos artefactos artísticos han despertado un gran interés en la crítica, que los ha estudiado desde múltiples perspectivas y que ha ofrecido, consecuentemente, numerosas definiciones más o menos afortunadas. La literatura sobre el tema obliga a Paz, en cierto modo, a ser preciso y claro desde el comienzo de su análisis. Sin rodeos ni divagaciones, ofrece su definición desde el inicio del párrafo:

Los ready-mades son objetos anónimos que el gesto gratuito del artista, por el solo hecho de escogerlos, convierte en obras de arte. Al mismo tiempo, ese gesto disuelve la noción de "objeto de arte". La contradicción es la esencia del acto; es el equivalente plástico del juego de palabras: este destruye el significado, aquel la idea de valor. Los ready-mades no son anti-arte, como tantas creaciones modernas, sino a-rtísticos. Ni arte ni anti-arte sino algo que está entre ambos, indiferente, en una zona vacía (1992b: 141).

Apenas se ha hecho alusión a la ironía antes del texto que citamos, pero de algún modo guarda un sorprendente parecido con la definición que otorga Kierkegaard de la ironía: “dejar solamente el vacío". Si bien, como ya hemos señalado anteriormente, en el pensamiento de Kierkegaard ese vacío tiene un fin cognoscitivo y teológico que, obviamente, no entra aquí en consideración. Al tratarse de objetos anónimos elevados a la categoría de obras de arte se produce 
un efecto contradictorio: la apariencia de dichos objetos no es comparable a la de ningún artefacto artístico y, de esa manera, atentan contra la noción misma de obra de arte. Se posicionan en un lugar que está más allá de la belleza y de la fealdad porque "no son obras sino signos de interrogación o de negación frente a las obras" (1992b: 142).

No se reduce a eso, por otro lado. Octavio Paz identifica dos etapas o dos críticas dentro de los ready-mades, lo que, en el fondo, viene a significar lo mismo que dos fases de la ironía: en un primer momento, estos objetos atentan contra la sensibilidad artística del impresionismo pictórico puesto que, a pesar de haber liberado formalmente el trazo para presentar una mirada subjetiva de la realidad, dicho movimiento repetía los mismos modos de significar que el arte figurativo anterior. Es decir, tan solo introducía un cambio de sensibilidad y de gusto: cambió las convenciones artísticas y no la concepción del arte. Duchamp se refería a estas formas de expresión como arte retiniano por vehicular un lenguaje exclusivamente visual cuyas formas significantes remitían directamente a unos significados concretos y hasta convencionales. A través del gesto gratuito del artista, Duchamp reduce la operación artística a su mínima expresión: de la fabricación al acto mismo, de la producción a la espectacularización.

El segundo momento del ready-made, según Paz, pasa de ser una crítica al gusto impresionista a ser una crítica del arte mismo; es decir, trasciende el contexto histórico para convertirse en una crítica no de la norma artística hegemónica sino del arte como fenómeno cultural. El giro que se produce desde la ironía hacia la metaironía se fundamenta, no exclusivamente pero sí en gran medida, en el salto que se produce desde una crítica estética del arte moderno a una crítica del arte como fenómeno o acontecimiento. En una suerte de juego de perspectivas y subversión de las categorías tradicionales del arte, Duchamp trasciende su propia tradición y observa, desde un afuera irónico, el acto de creación y de contemplación de la obra. ¿Cómo se produce la metaironía? A través de una crítica a la significación artística:

Las significaciones de la pintura "retiniana" son insignificantes: impresiones, sensaciones, secreciones, eyaculaciones. El ready-made enfrenta a esta insignificancia su neutralidad, su no-significación. Por tal razón no debe ser un objeto hermoso, agradable, repulsivo o siquiera interesante. Nada más difícil que encontrar un objeto realmente neutro [...]. Desalojado, fuera de su contexto original -la utilidad, la propaganda o el adorno- el ready-made pierde bruscamente todo significado y se transforma en un objeto vacío, en cosa en bruto (1992b: 143).

En el ready-made la selección del objeto, junto con el título de la obra, es lo único que determina el modo en que se produce finalmente la ironía. La obra de arte es auténtica y única, como bien advirtió Walter Benjamin (1892-1940) en su obra La obra de arte en la época de su reproductibilidad técnica (1936); por 
muy precisa que pretenda ser su reproducción, la obra está dotada de la autenticidad que le confiere el aquí y el ahora (Benjamin, 2018: 198). Dicha cualidad abstracta toma el nombre de aura. ¿Cómo se puede sortear el aura de la obra, que al fin es lo que pretende romper Duchamp con su crítica, si es un objeto único e irreproducible? La negación de su autenticidad se encuentra, como es lógico, en la propia selección del objeto. Duchamp selecciona los objetos por su fabricación industrial, aquella que se sirve de técnicas concretas para producir objetos idénticos en masa, con el fin de negar la utilidad de los mismos. Al tiempo que niega la obra de arte, se enfrenta a la utilidad con la que dichos objetos fueron concebidos puesto que los despoja de su cualidad esencial.

Estas razones son las que nos permiten afirmar que los ready-mades se posicionan en el extremo diametralmente opuesto de la crítica benjaminiana: no es la reproducción técnica de la obra de arte sino la obra de arte convertida en reproducción técnica o, mejor dicho, la reproducción técnica elevada a la categoría de arte. Sin embargo, lejos de buscar la abolición del aura artística en sí misma, su objetivo es eliminar la amenaza del mercantilismo.

Lejos de un homenaje a la era industrial, pulsión frecuente en las vanguardias, el artista critica la ambivalencia de la era moderna: la progresiva divinización -y mercantilización- del objeto artístico, y la progresiva desvalorización de los objetos producidos en masa gracias a las nuevas técnicas industriales. Como advertiría el propio Octavio Paz, gran parte de la obra duchampiana consistiría en la abolición del propio mito de su obra: "Duchamp [...] juega al ajedrez mientras yo escribo estas notas" (1992b: 143). La crítica a la producción asume múltiples formas en Duchamp, pero una de las más radicales es el abandono de la creación artística para dedicarse únicamente al ajedrez - por supuesto, nunca fue realmente así y su obra continuó varios años en secreto. Tras abolir el aura del objeto artístico, tan solo quedaría por abolir el aura del propio artista, y Duchamp lo haría renunciando primero a la pintura y luego al arte durante décadas. La idoneidad de la crítica artística de Walter Benjamin para comprender la obra de Duchamp consiste en un cierto parentesco, aunque provenga de razones casi contrarias: si bien el primero parte de una crítica a la producción capitalista desde una perspectiva marxista, el segundo, sin marcas ideológicas, critica dicha producción llevando a cabo un uso irónico de la misma. Esta es la dirección que toma el maquinismo en la obra del artista francés; es un componente de la ironía crítica, pero sobre ello nos detendremos en el próximo epígrafe y no en este.

Octavio Paz rara vez nunca se sirvió de la retórica marxista y revolucionaria para elevar una crítica de su tiempo. Si bien tenemos constancia de esta tendencia ideológica en su juventud, en su madurez encontramos, más bien, cierta equidistancia hacia los dos bloques políticos internacionales que protagonizaron la Guerra Fría. Su proyecto intelectual y literario se impuso, por así decirlo, sobre su proyecto político; sin embargo, en las últimas décadas de su producción se acentuó considerablemente su crítica al uso que el sistema capitalista había hecho del cuerpo y del erotismo. 
Donde más claramente puede observarse esta crítica es en su libro La llama doble (1993), pero también se puede percibir, por ejemplo, en el análisis que realiza del pensamiento de Charles Fourier (1772-1837) en Los hijos del limo, y otros textos. La liberación erótica y de los cuerpos, que tanta importancia tendría en el surrealismo de André Breton (1896-1966) y la revolución sexual, constituiría un núcleo temático fundamental en la obra de $\mathrm{Paz}^{5}$. Advertimos la importancia del erotismo por la correspondiente aplicación que va a tener en el próximo epígrafe, y también por su presencia en los ready-mades. Su análisis de los mismos se cierra en El castillo de la pureza, antes de adentrarse en el desciframiento del lenguaje del Gran vidrio, con un último párrafo donde compara el acto de escoger el objeto con una cita. Una cita, un encuentro de realidades, sí, pero irónica y desprovista de erotismo: "es una cita con nadie y su finalidad es la no-contemplación [...]. Entronizar una nadería y, una vez en su trono, negarla y negarse a sí mismo" (Paz, 1992b: 148).

\section{CONCLUSIONES}

En este trabajo de investigación nos hemos aproximado a algunos conceptos fundamentales del pensamiento poético y filosófico de Octavio Paz a partir de sus reflexiones sobre la obra artística temprana de Marcel Duchamp, concretamente, aquellos objetos que eran propios de la era industrial y que fueron nombrados ready-mades. El escrito mexicano había construido ya para los años 60 ' un armazón teórico sólido a través de obras tan importantes como El laberinto de la soledad (1950) o El arco y la lira (1956), que si bien constituían ensayos más o menos novedosos servían para enmarca el pensamiento de su autor en una tradición concreta, presentando todos ellos una interpretación personal de la tradición literaria occidental. Estos estudios, sin embargo, se adelantaban de alguna manera y no se verían culminados teóricamente hasta su descripción crítica de la modernidad poética en Los hijos del limo (1974), apenas unos años después de que publicara la primera versión de El castillo de la pureza (1966). Esto nos mueve a pensar que la escritura, sino contemporánea al menos mantuvo una cierta continuidad teórica.

De entre los muchos críticos de arte que se aproximaron antes y después que él, Octavio Paz destaca como uno de los más lúcidos y originales, puesto que no se trataba de una simple reflexión en torno a una obra de arte. Sus reflexiones son eslabones de un armazón teórico desarrollado durante décadas y que encajan como piezas de una maquinaria industrial dentro del camino prediseñado de la modernidad. Como un camino cuyos recodos y giros fueran, en sí mismos, necesarias inflexiones para alcanzar la meta. Como mostrando una cierta

\footnotetext{
${ }^{5}$ Las referencias que al erotismo hace en El castillo de la pureza aparecían en la edición de 1968, por lo que la preocupación filosófica que muestra Octavio Paz por el erotismo es varias décadas anterior a la escritura de La llama doble (1993).
} 
lógica de la superación dentro de la modernidad, y que desembocaría en otra cosa, en otra época. El procedimiento de tal superación, dondequiera que desemboque, es la ironía que se deslinda de la continuidad y de la discontinuidad, o de la otredad y de la identidad. Se trata de un ejercicio de toma de consciencia y de pertenencia, de tomar distancia con respecto a la tradición propia con el fin de criticarla, de trascenderla y abrir nuevos caminos para la creación. Exactamente como hicieron ambas figuras, Paz y Duchamp, a través de los años en sus reflexiones teóricas y sus creaciones estéticas.

\section{BIBLIOGRAFÍA}

BALLART, P. (1994): Eironeia. La figuración irónica en el discurso literario moderno. Barcelona: Quaderns Crema.

BAUDELAIRE, C. (1995): El pintor de la vida moderna. Murcia: Cajamurcia.

BENJAMIN, W. (2018): Iluminaciones. Madrid: Taurus.

CERVERA, V. (2001): La poesía y la idea. Fragmentos de una vieja querella. Murcia: Editum.

DUCHAMP, M. (2016): Cartas sobre el arte (1916-1956). Barcelona: Elba.

FONG, M. (2014): Entre Octavio Paz et Marcel Duchamp. París: L'Échoppe.

HÖLDERLIN, F. (2014): Principis per a la consecució poètica. Barcelona: Quaderns Crema.

(1976): Ensayos. Madrid: Hiperión.

NOVALIS (1988): Los discípulos de Sais. Madrid: Hiperión.

PAZ, O. (1999): La casa de la Presencia: Poesía e historia (vol. I). Barcelona: Galaxia Gutenberg.

(1992b): Los privilegios de la vista I (vol. IV). Barcelona: Círculo de Lectores.

VENTURA RAMOS, L. (2015): “Ironía romántica: un principio paradójico de representación literaria”. Tópicos del seminario: núm. 34, págs. 83-106. 